\title{
Reshaping of Large Aeronautical Structural Parts: A Simplified Simulation Approach
}

\author{
Ramiro Mena ${ }^{1, a)}$, José V. Aguado ${ }^{2}$, Stéphane Guinard ${ }^{1}$ and Antonio Huerta ${ }^{3}$ \\ ${ }^{1}$ Airbus Group Innovations, \\ 18, rue Marius Terce, 31300 Toulouse, France \\ ${ }^{2}$ ICI-HPC Institute at Ecole Centrale de Nantes, \\ 1 Rue de la Nö̈, 44321 Nantes, France \\ ${ }^{3}$ Laboratori de Calcul Numeric (LaCaN). Departament de Matematica Aplicada III. E.T.S. de Ingenieros de \\ Caminos, Canales y Puertos, Universitat Politecnica de Catalunya, BarcelonaTech, \\ 08034 Barcelona, Spain. \\ a) Corresponding author: ramiro-francisco.mena-andrade@ airbus.com
}

\begin{abstract}
Large aeronautical structural parts present important distortions after machining. This problem is caused by the presence of residual stresses, which are developed during previous manufacturing steps (quenching). Before being put into service, the nominal geometry is restored by means of mechanical methods. This operation is called reshaping and exclusively depends on the skills of a well-trained and experienced operator. Moreover, this procedure is time consuming and nowadays, it is only based on a trial and error approach. Therefore, there is a need at industrial level to solve this problem with the support of numerical simulation tools. By using a simplification hypothesis, it was found that the springback phenomenon behaves linearly and it allows developing a strategy to implement reshaping at an industrial level.
\end{abstract}

\section{INTRODUCTION}

In the aircraft industry, the technical requirement of minimum weight with maximum strength generates parts with complex geometries. As a result, large monolithic structures are made of aluminum forgings. These components present residual stresses as a consequence of non-uniform plastic strains generated during previous manufacturing steps, especially after quenching ${ }^{1,2}$. When parts are machined, in order to obtain the final geometry, almost $90 \%$ of material is removed under high feeds and high cutting speeds. This material removal brakes the previous stable configuration and a redistribution of residual stresses take place ${ }^{3}$. As a result, important distortions that are out of the geometrical tolerances arise. Before being put into service, distortions are removed manually by mechanical methods. This operation is called reshaping and exclusively depends on the skills of a well-trained and experienced operator. Moreover, this procedure is time consuming and nowadays, it is only based on a trial and error approach. Therefore, there is a need at industrial level to solve this problem with the support of numerical simulation tools ${ }^{4}$.

Reshaping can be interpreted as an optimization problem, where the goal is to introduce the minimum number of extra reshaping steps in order to fit the geometry inside a given tolerance. The idea behind this post-machining operation is to produce localized plastic strains in determined part's zones to minimize remaining distortion. One common reshaping operation consists in loading the distorted part backward (bending in the opposite direction to observed distortions): during unloading, as the external load is removed, the elastic component of strain is released and the springback phenomenon is present. As a consequence, an extra plastic strain is required to compensate this issue. Although it was originally applied to straightening of seam pipes, Katoh et al., proposed to approximate the real springback behavior by a linear hypothesis in a force-displacement diagram ${ }^{5}$. This idea is translated to our 
problem and tested for two different geometries: a rectangular beam and a $\mathrm{T}$ shaped beam. The first case is solved experimentally to validate the range of applicability of Katoh's hypothesis, whereas the second case is solved numerically. This last model corresponds to a simplified version of a cruciform part, which exhibits critical distortions when machined and is a primary structure of main importance for aircraft manufacturers.

This document is organized as follows: first, the idea of the force displacement diagram applied to reshaping is explained. Then, the problem setup used for the two beam geometries is described. Next, the results of both experiments are analyzed and finally, the obtained conclusions are addressed.

\section{FORCE DISPLACEMENT DIAGRAM}

Given an initial distortion, a warped part is straightened by using mechanical operations, normally a three or four point bending. The first case is selected for localized distortions such as kinks whereas the last is used when distortion is progressively distributed along the part. For the rest of the study, the four point bending is chosen and it is represented in Fig. 1 (a), where $L$ defines the effective span length, $\delta_{r}$ corresponds to the reference distortion and $a$ is the distance between roller supports and the applied force $F$.

For a defined technological setup (i.e roller positioning), there is an imposed given displacement, called stroke $Y_{s}$, capable to minimize distortion, where the springback $\delta_{s}$ is properly compensated. In order to find this configuration, a traditional FEM algorithm obliges to run a new simulation each time $Y_{s}$ is modified. A more suitable approach, proposed by Katoh et al., used the force $F$ - displacement $\delta$ diagram to compensate in real-time the springback behavior by assuming that the gradients during loading and unloading are parallel ${ }^{5}$. It means that the equivalent Young's modulus $\mathrm{E}_{0}$, calculated during loading, can be used for unloading $\hat{\mathrm{E}}_{0}, \mathrm{E}_{0} \approx \hat{\mathrm{E}}_{0}$. Therefore, as $\delta_{r}$ is an initial data and corresponds to the distortion to be removed, the loading stage during reshaping will take place until the resultant $F$ - $\delta$ curve intersects the predicted unloading path.

In the $F$ - $\delta$ diagram (Fig. 1 (b)), during loading, the slope of the curve is defined by $E_{0}$, and follows the relation:

$$
F=E_{0} \delta
$$

For the selected reshaping operation, $E_{0}$ can be found theoretically by using the beam expression:

$$
E_{0}=24 E I /\left[\left(3 L^{2}-4 a^{2}\right) a\right]
$$

where $E$ and $I$ are the Young's modulus and inertia of the cross section, respectively. Another option is, given two load and displacement points before yielding, named as $\left(F_{1}, \delta_{l}\right)$ and $\left(F_{2}, \delta_{2}\right)$, respectively, $\mathrm{E}_{0}$ can be determined experimentally as:

$$
E_{0}=\left(F_{2}-F_{1}\right) /\left(\delta_{2}-\delta_{1}\right)
$$

this last approach has the advantage that no previous material modeling information is needed. Additionally, when it comes to a part with residual stresses, it allows overcoming the uncertainties associated with simulating each manufacturing step, simplifying the problem and adapting the response to each specific part.

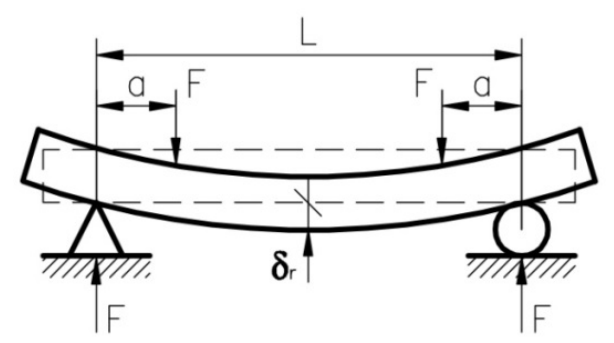

(a)

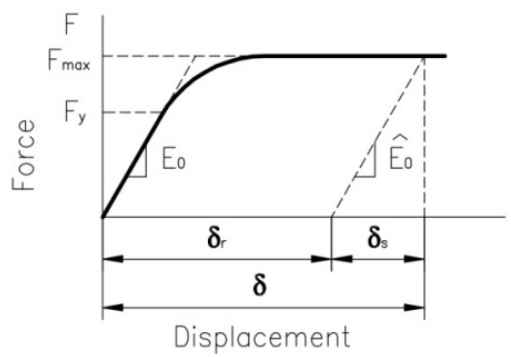

(b)

FIGURE 1. Reshaping operation. (a) Initial warped geometry, (b) Force vs displacement diagram. 


\section{PROBLEM SETUP}

In order to study reshaping, two models are proposed. First, starting from a flat configuration, a rectangular beam with chamfers at the top surface is plastically deformed by imposing a vertical displacement of $\pm 9,5 \mathrm{~mm}$ during three cycles. The sample's geometry and the test setup are represented at Fig. 2 (a) and (b), respectively. For this test, displacements are recorded at two locations: at the beam's mid-span and where the force is applied. Additionally, the force data acquisition is done by the hydraulic press' head and strain data at the top and bottom faces are recorded by two strain gauges, located at the intersection of both longitudinal and transversal symmetry planes of the beam. The goal of this test is to validate the applicability of Katoh's hypothesis for a simple geometry subjected to a deformation in the range of $4 \%$.

The second model corresponds to a $\mathrm{T}$ shaped beam. This case is based on the results previously obtained in reference ${ }^{6}$, where distortion was generated numerically and two configurations were selected, named as configuration A and B, respectively (see Fig. 3 (c)). Then, reshaping was done by incrementing sequentially the imposed vertical stroke $Y s$ until distortion was minimized. The results were calculated by using a nonlinear material behavior. Now, they are compared by assuming an unloading linear path.

For both experiments, the selected material is aluminum AA7010, which was previously characterized by Airbus. Its elastic properties are summarized in Table 1. To run the reshaping simulations, a Chaboche's isotropic/nonlinear kinematic hardening model was used. However, as the material parameters are under a nondisclosure agreement, they are not included in this paper.

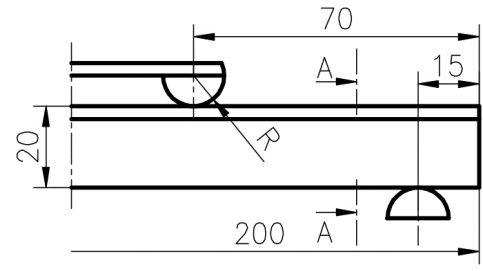

(a)

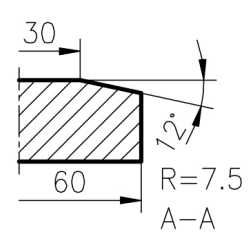

-A

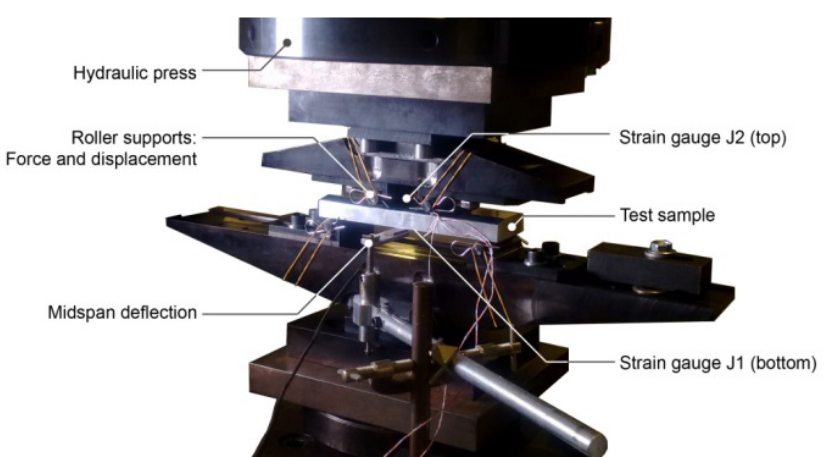

(b)

FIGURE 2. Rectangular beam experiment. (a) Geometry definition, (b) Laboratory setup.

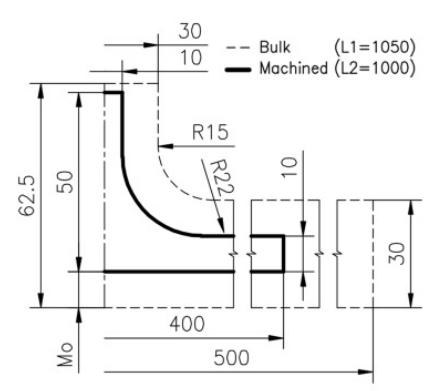

(a)

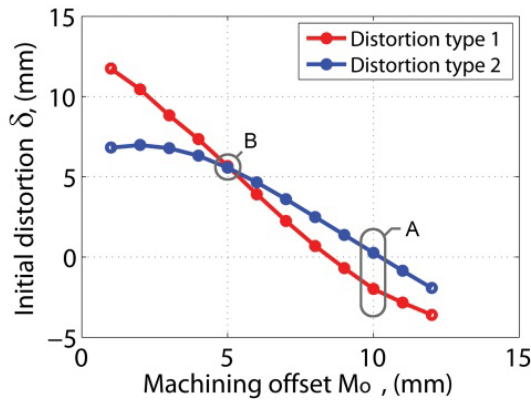

(b)

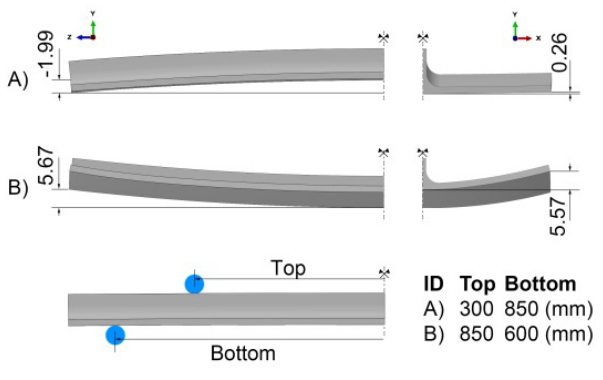

(c)

FIGURE 3. T shaped beam model. (a) Geometry definition, (b) Initial distortion as a function of machining offset $M_{O}$, (c) Distorted geometry cases and reshaping setup.

TABLE 1. Elastic properties for Aluminum AA7010

\begin{tabular}{ccc}
\hline Young's Modulus E $(\mathbf{M P a})$ & Poisson's ratio $v(-)$ & Yield stress $\sigma_{\mathbf{y}}(\mathbf{M P a})$ \\
\hline 73000 & 0,3 & 390 \\
\hline
\end{tabular}




\section{RESULTS AND DISCUSSION}

For the first experiment, the force-displacement diagram is obtained at two locations: the beam's mid-span and the section where the imposed displacement is applied. Both diagrams are represented in Fig. 4 (a) and (b), respectively. The equivalent Young's modulus $\mathrm{E}_{0}$ is calculated by using equation (3), then, a linear unloading path is represented at the final position of each cycle. It can be seen that the experimental unloading path presents a small discontinuity at the beginning and then, it follows a nonlinear trajectory. However, this trajectory is convex and for reshaping, we are interested in two points: where to stop the loading curve and what is the final distortion in the part. Therefore, if the linear approximation of the unloading path is used, both points can be tracked in a straightforward way for a given tolerance.

An explanation to Katoh's hypothesis can be found in literature ${ }^{7}$, where Yu et al., state "When a complete unloading is performed after applying a bending moment $M$ to a beam, if unloading does not cause reverse yielding, then effectively the unloading process is equivalent to the elastic effect caused by applying - $M$ to the beam". Here, the key is to not produce reverse yielding. This phenomenon is present in the automotive industry during metal sheet forming. However, the structural elements that are subjected to reshaping in the aircraft industry can be considered as thick walled, and the levels of strain under reshaping cannot produce reverse yielding. Therefore, the unloading path can be approximated to a linear behavior. A similar analogy can be done respect to the residual stresses generated during machining, which are required to be included in a distortion simulation for a thin walled component $^{8}$ or that can be neglected in a thick walled part ${ }^{9}$.

If the lineal hypothesis is valid during unloading, then superposition can be used and the residual stresses $\sigma_{\text {residual }}$ can be found by adding the stresses generated during loading $\sigma_{\text {load }}$ to the unloading stresses $\sigma_{\text {unload}}$ :

$$
\sigma_{\text {residual }}=\sigma_{\text {load }}+\sigma_{\text {unload }}
$$

By reproducing numerically the experiment, for the values obtained during the $1^{\text {st }}$ cycle, $\sigma_{\text {load }}$ and $\sigma_{\text {residual }}$ are known, which correspond to the stress states at the beginning and at the end of the unloading step, respectively. Then, $\sigma_{\text {unload }}$ is obtained by using expression (4). The previous stress states are shown in Fig. 5 and they are represented along the cross section height (y-axis) at the intersection of the longitudinal and transversal symmetry planes. Again, it can be seen how $\sigma_{\text {unload }}$ presents a nonlinear distribution (Fig. 5 (b)). When fitted linearly, an approximated residual stress distribution is obtained (Fig. 5 (c) in dashed lines), which provides a close approximation of the result obtained numerically. After each cycle, the residual stress (RS) follows a triple zerocross pattern ${ }^{10}$, its order of magnitude is around $\pm 200 \mathrm{MPa}$ after unloading and bend straightening causes RS similar to the values generated after quenching.

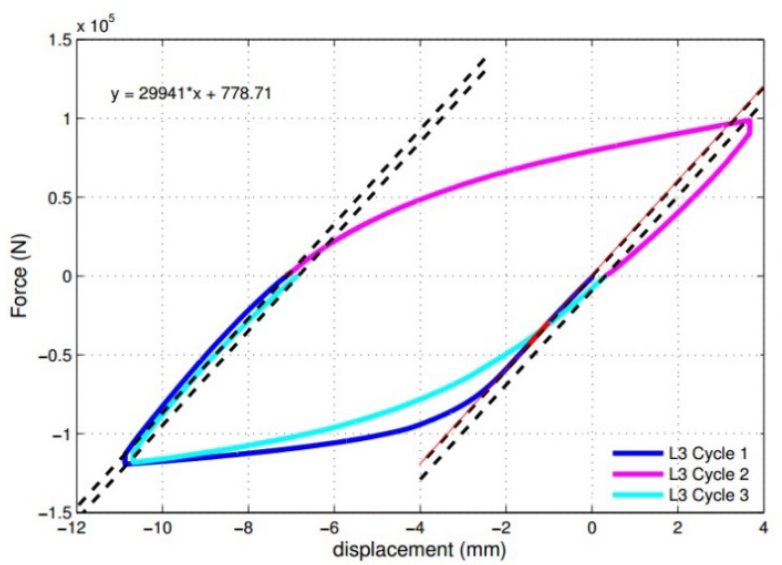

(a)

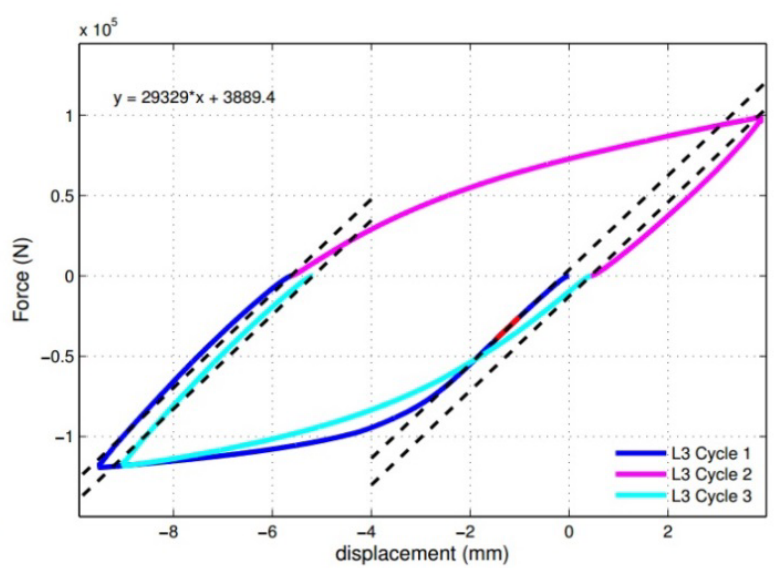

(b)

FIGURE 4. Force vs displacement diagram for the rectangular beam. Experimental curves (solid lines) vs linear unloading path (dashed lines). (a) Mid-spam L=100 and (b) Imposed stroke $Y s= \pm 9,5 \mathrm{~mm}$. 
For the second experiment, corresponding to the $\mathrm{T}$ shaped beam, the numerical results were obtained in a previous work with a nonlinear material model ${ }^{6}$. A similar procedure as the one previously described is used to calculate $\mathrm{E}_{0}$. Again, a force-displacement diagram is generated for reshaping configurations $\mathrm{A}$ and $\mathrm{B}$, which are represented in Fig. 6 (a) and (b), respectively. As it can be seen, the linear unloading path approximation is valid for both reshaping configurations, although, configuration B presents a more pronounced nonlinear unloading path, probably caused by the higher initial distortion. From the multiple available stroke scenarios, we are only interested in the parameter that minimizes distortion, which corresponds to the red curves depicted in both figures. For the optimum curves, if the linear unloading path is used, the difference in terms of maximum load during reshaping is less than 3\%. The corresponding values for configuration A and B are summarized in Table 2. Additionally, if both configurations are considered as the extreme values for the interval of machining offset $M o=[5,10]$ in the initial distortion diagram (see Fig. 3 (b)), it is expected that Katoh's hypothesis remains valid for intermediate values as well.

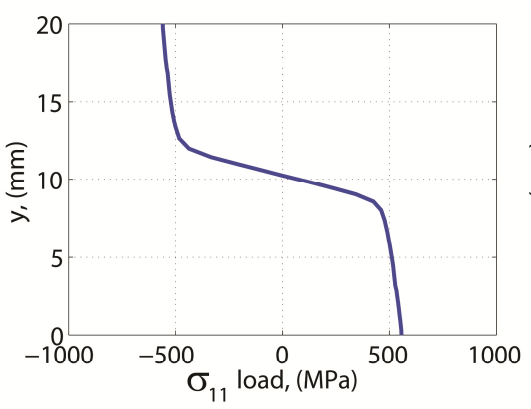

(a)

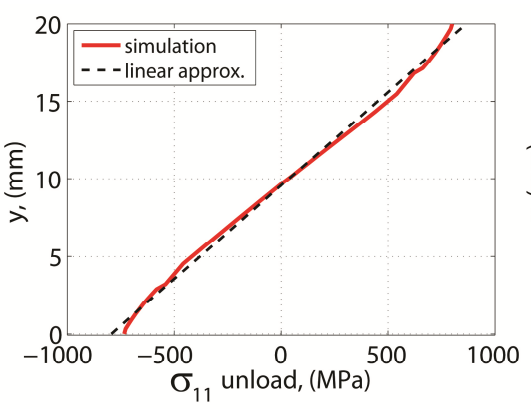

(b)

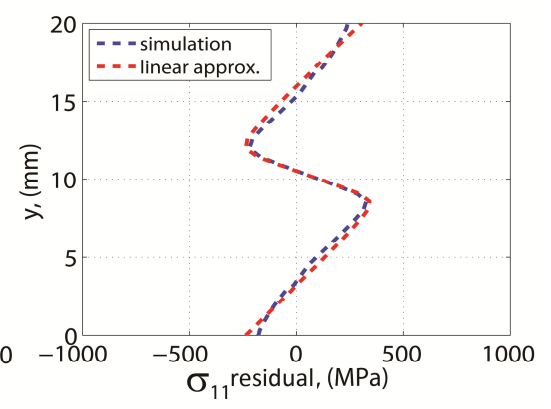

(c)

FIGURE 5. Longitudinal stress $\sigma_{11}$ for the rectangular beam. (a) Loading, (b) Unloading and (c) Residual

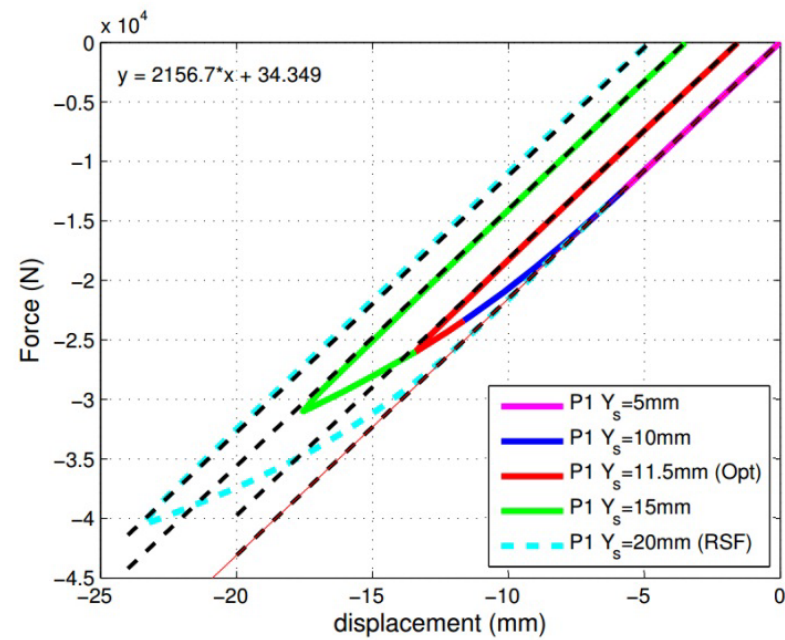

(a)

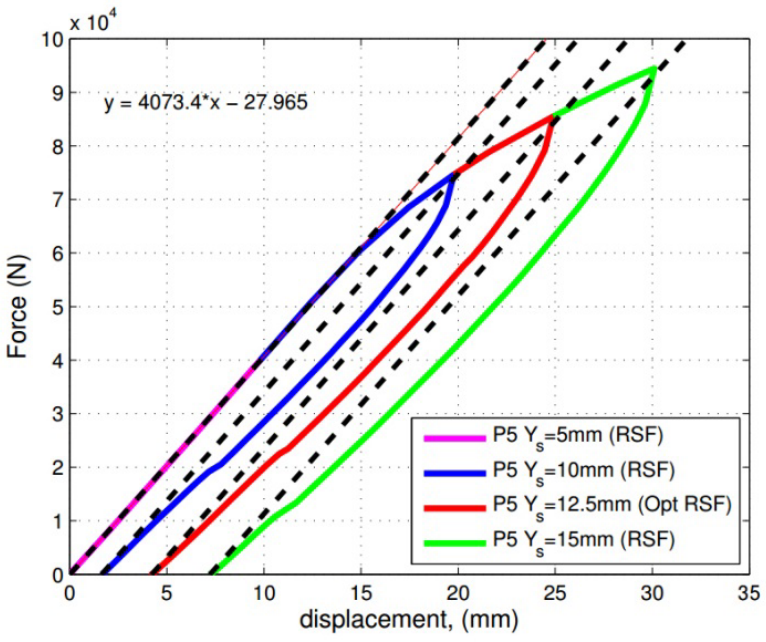

(b)

FIGURE 6. Force vs displacement diagram for the $T$ shaped beam. Numerical curves (solid lines) vs linear unloading path (black dashed lines). (a) Reshaping configuration A and (b) Reshaping configuration B.

TABLE 2. Starting point for the unloading curve at the optimum stroke (named as Opt in Fig. 6)

\begin{tabular}{cccc}
\hline Configuration & Numerical max. load $(\mathbf{K N})$ & Simplified max. load $($ KN) & Error $(\%)$ \\
\hline A & -25.99 & -26.7 & 2.732 \\
B & 85.5 & 86.52 & 1.193 \\
\hline
\end{tabular}




\section{CONCLUSIONS}

Reshaping simulations can be considered computationally expensive, as 3D solid elements are used and multiple simulation steps are required before obtaining an initial warped geometry. Additionally, an adaptive response is desired to modify a simulation if some reshaping conditions are changed at the workshop level. Therefore, some simplifications hypotheses are needed in order to implement the numerical results in an industrial environment. Following this line, the idea of considering a distorted part free of residual stresses for simulating reshaping was able to describe the part's distortion evolution compared to the case with residual stresses ${ }^{6}$, however, its resolution remains subjected to the progressively variation of the imposed vertical stroke $Y s$ until distortion $\delta$ is minimized. Now, by using a linear approximation to the unloading path, only one loading simulation would be required and multiple unloading scenarios can be easily explored. Finally, the force and displacement measurement are parameters that can be implemented in an industrial environment.

\section{ACKNOWLEDGMENTS}

This project has received funding from the European Union's Horizon 2020 research and innovation programme under the Marie Skłodowska-Curie grant agreement No 675919.

\section{REFERENCES}

1. P. Jeanmart, J. Bouvaist, Mat. Sci. Tech. 1, 765-769 (1985).

2. D.A. Tanner, J.S. Robinson, Finite Elem. Anal. Des. 39, 369-386 (2003).

3. J.S. Robinson, D.A. Tanner, C.E. Truman and R.Wimporoy, Exp. Mech 51, 981-993 (2011).

4. W.-M. Sim, "Challenges of Residual Stress and Part Distortion in the civil airframe industry", in $2^{\text {nd }}$ International Conference on Distortion Engineering (IDE)-2008, pp. 87-94.

5. T. Katoh, E. Urata, M. Nakanishi and K. Yamazaki, JSME. 34, 427-432 (1991).

6. R. Mena, D. Deloison, J.V. Aguado, and A. Huerta, "Influence of the residual stresses in reshaping operations of large aeronautical parts," in International Conference on Adaptative Modeling and Simulation ADMOS 2017, edited by S. Perotto and P.Díez, eds., CIMNE, 2017.

7. T.X. Yu and L.C Zhang, Plastic Bending: Theory and applications, (World Scientific, Singapore, 1996), pp. 20

8. K. Ma, R. Goetz and S.K.Srivatsa, Modeling of residual stress and machining distortion in aerospace components (Air Force Research Laboratory, Ohio, 2010), pp. 3.

9. X. Cerutti, "Numerical modelling and mechanical analysis of the machining of large aeronautical pats: Machining quality improvements,” Ph.D. thesis, Ecole Nationale Supérieure des Mines de Paris, 2014.

10. A.Ellerman and B.Scholtes, Int. J. Mater. Res 103, 57-65 (2012). 